\title{
Management of postmenopausal osteoporosis
}

\author{
Yeap SS, Hew FL, Chan SP, on behalf of the Malaysian Osteoporosis Society Committee \\ Working Group for the Clinical Guidance on the Management of Osteoporosis, 2012
}

Yeap SS, Hew FL, Chan SP, on behalf of the Malaysian Osteoporosis Society Committee Working Group for the Clinical Guidance on the Management of Osteoporosis, 2012 Management of postmenopausal osteoporosis. Malaysian Family Physician 2013;8(2): 36-40

\section{Keywords \\ osteoporosis, \\ treatment, \\ Malaysia, \\ postmenopausal, \\ calcium, \\ diagnosis}

\section{Authors}

\section{Yeap Swan Sim MBChB (Dundee), MD (Dundee), FRCP (Edin), FRCP (Lond), CCST (UK) \\ (corresponding author) Subang Jaya Medical Centre, \\ 47500 Subang Jaya, Selangor,Malaysia Tel: +60356391212 Fax: +60356391319 Email: yeapss@myjaring.net}

Hew Fen Lee MBBS (Newcastle), MD (Melbourne), FRCP (Edin), FRACP

Subang Jaya Medical Centre,

47500 Subang Jaya, Selangor, Malaysia

Chan Siew Pheng MBBS (Mal), FRCP (Edin) Subang Jaya Medical Centre,

47500 Subang Jaya, Selangor, Malaysia

\section{Epidemiology}

Osteoporosis (OP) is a systemic skeletal disease characterised by low bone mass and microarchitectural deterioration of bone tissue, with a consequent increase in bone fragility and susceptibility to fracture. Common sites of osteoporotic fractures are in the spine (vertebral), hip (proximal femur) or wrist (distal forearm). In Malaysia, in 1997, the incidence of hip fracture among individuals above 50 years of age was 90 per 100,000 population. The incidence increasedwith age; in the 50-54 year olds, the incidence was 10 per 100,000 , rising to 510 per 100,000 in those over 75 years old.

OP fractures are associated with significant morbidity and mortality. Following a hip fracture, there is a reduction in survival of 10$20 \%$ over next 1 year. For clinically diagnosed vertebral fractures, there is a relative survival rate of 0.82 (18\% reduction) at 5 years following the diagnosis.

\section{Diagnosis}

The risk factors for OP are shown in Table 1. However, the use of risk factors have not been shown to predict bone mass with sufficient precision to be useful in the assessment of fracture risk or bone mineral density (BMD) of individuals. It is thus better to measure BMD, of which the gold standard is using dualenergy x-ray absorptiometry (DXA). DXA measurement also allows the classification of OP which is defined by a BMD of more than -2.5 standard deviation (SD) from the young adult mean (T-score) and osteopenia when the $\mathrm{T}$-score is between -1.0 and -2.5 . BMD measurement is recommended in women aged
65 and older, and men aged 70 and older. In postmenopausal women and men aged 50-69, BMD measurement is recommended in the presence of strong risk factors and if the results will influence management.

Fracture risk approximately doubles for each SD decrease in BMD. Age is a major risk factor for OP fracture independent of BMD. At any level of $B M D$, current fracture risk is low in the 50-54 year olds, with an exponential increase with age. For example, the 10 year fracture probability is approximately $10 \%$ at a $\mathrm{T}$-score of -2.5 at the age of 50 , but increases to $25 \%$ at the age of 80 . A history of previous OP fracture also predicts future fracture independent of BMD. Women with pre-existing vertebral fractures (identified at baseline by vertebral morphometry) had approximately 4 times greater risk of subsequent vertebral fractures than those without prior fractures. Overall, those with prior fractures had $2.2(95 \% \mathrm{CI}=$ 1.9, 2.6) times the risk of subsequent fracture compared with those without prior fractures.

Therefore, when a patient presents with a low trauma fracture, osteoporosis is a presumptive diagnosis. BMD measurement with DXA is advised. However, in the absence of this facility, treatment should still be initiated.

The Fracture Risk Assessment Tool (FRAX) (www.shef.ac.uk/frax) estimates the 10year probability of hip fracture and major osteoporotic fracture (hip, clinical spine, proximal humerus, or forearm), for untreated patients between ages 40 to 90 years using clinical risk factors. BMD is not necessary for calculation of fracture probability. However, it 
improves the prediction of fracture probability. If a BMD is available, only the femoral neck/ total hip BMD is to be used. BMD input from non-hip sites has not been validated with FRAX and is therefore not recommended. The Singapore prediction algorithm should be used

Table 1. Risk factors for osteoporosis

\section{Non-modifiable}

\author{
1. Advancing age \\ 2. Ethnic group (Oriental \& Caucasian) \\ 3. Female gender \\ 4. Premature menopause ( $<45$ years) \\ including surgical menopause \\ 5. Family history of osteoporosis or fracture \\ in first degree relative \\ 6. Personal history of fracture as an adult
}

when using the FRAX tool.

Quantitative ultrasound (QUS) in the diagnosis of $\mathrm{OP}$ and monitoring of treatment is not recommended.

\section{Non-pharmacological Treatments}

A balanced diet with adequate protein, calcium and vitamin $\mathrm{D}$ is important for bone health. For women and men over the age of 50, the daily recommended calcium intake is $1000 \mathrm{mg}$, which includes both dietary and supplemental calcium. The absorption of calcium supplements is highly variable ranging from $20-40 \%$ depending on the formulation as shown in Table 2. For optimal absorption, the amount of calcium should not exceed 500 to $600 \mathrm{mg}$ per dose, irrespective of the calcium preparation. For patients requiring more than $600 \mathrm{mg}$ of calcium supplement daily, the dose should be divided.

There is controversy whether calcium supplementation can lead to increased cardiovascular (CV) events. There have been studies both showing an increased risk of myocardial infarction, and others showing no excess CV mortality with calcium supplements. The excess CV risk was not reduced by the addition of vitamin $\mathrm{D}$ supplementation. The latest prospective study published in February 2013 showed no excess of all causes of mortality with calcium intakes of $<1400 \mathrm{mg}$ daily. Calcium-rich foods have not been associated with a higher risk of coronary heart disease. We therefore recommend a maximum total daily calcium intake of $1000 \mathrm{mg}$, both from dietary sources and supplements.

It is recommended that vitamin D intake is 800 IU daily. Blood levels of $25(\mathrm{OH}) \mathrm{D}$ provide the best index of vitamin $\mathrm{D}$ stores. It has been suggested that levels of $25(\mathrm{OH}) \mathrm{D}$ of $>20 \mathrm{ng} /$ $\mathrm{ml}(50 \mathrm{nmol} / \mathrm{l})$ are adequate for skeletal health.

Following an OP fracture, all patients should have a fall assessment for prevention of future falls. They should be advised on increasing exercise and reducing alcohol intake and smoking. 
Table 2. Calcium absorption from different sources

\begin{tabular}{lll} 
Type & $\begin{array}{l}\text { Elemental Calcium (\%) } \\
\text { Calcium carbonate }\end{array}$ & $\begin{array}{l}\text { Average calcium absorption (\%) } \\
\text { (Range) }\end{array}$ \\
Calcium citrate & 40 & $26(13.8-64)$ \\
Calcium lactate & 21 & $22(12.3-31.4)$ \\
Calcium gluconate & 13 & 32 \\
\hline Milk (non-calcium enriched) & 9 & $34(21.8-67.5)$ \\
\hline
\end{tabular}

\section{Pharmacological Treatment}

The risk factors for OP are shown in Table 1. However, the use of risk factors have not been shown to predict bone mass with sufficient precision to be useful in the assessment of fracture risk or bone mineral density (BMD) of Following a clinical assessment, all patients should be advised on general measures to improve bone health. In patients who have already had a prior low trauma/fragility fracture, without any cause to suggest secondary OP, treatment can be started; measurement of BMD is ideal if available. For those with risk factors but no prior fracture, assessment using the FRAX tool is suggested. This can be used with or without a BMD measurement. In patients found to be have a high 10- year risk of fracture with FRAX, treatment should be started. In patients with a low risk of fracture, BMD can be measured and monitored at 1-2 yearly intervals. If FRAX is not available, and there is still concern about the possibility of OP, a BMD measurement can be obtained. Patients with a $\mathrm{T}$ score $\leq-2.5$ have $\mathrm{OP}$ and should be started on treatment. Patients with osteopenia ( $\mathrm{T}$-score between -1.0 and -2.5) can be started on treatment when the 10-year fracture probability is more than 3\% for hip and more than $20 \%$ for major osteoporosis-related fractures. Alternatively, if a FRAX assessment is not available but there are multiple risk factors present, treatment can be also started. Patients with a normal T-score (>-1.0) can be monitored with 1-2 yearly BMD measurements. Currently available treatments for OP are shown in Table 3. The suggested pathway for the treatment of postmenopausal OP is shown in Figure One. The choice of drug for established OP, especially those with a previous fracture, must be an agent shown not only to increase BMD, but also shown to reduce fracture both at the spine and hip.

Table 3. Efficacy of treatments available for osteoporosis

All the below treatments have been shown in randomised controlled trials to increase bone mineral density (Grade A).

\begin{tabular}{lll} 
Intervention & $\begin{array}{l}\text { Decrease Vertebral } \\
\text { Fracture Rate }\end{array}$ & $\begin{array}{l}\text { Decrease Hip Fracture } \\
\text { Rate }\end{array}$ \\
\hline Alendronate & A & A \\
Ibandronate & A $^{*}$ & - \\
\hline Risedronate & A & A \\
Zoledronate & A & A \\
\hline Strontium Ranelate & A & A \\
\hline Denosumab & A & A \\
\hline rPTH (teriparatide) & A & - \\
\hline Hormone Therapy & A & A \\
\hline Tibolone & A & - \\
\hline Raloxifene & A & - \\
\hline Calcitonin & A & - \\
\hline Calcitriol/Alfacalcidol & A & - \\
\hline Calcium \pm vitamin D & A & A \\
\hline
\end{tabular}


Figure 1. Algorithm for the management of postmenopausal osteoporosis

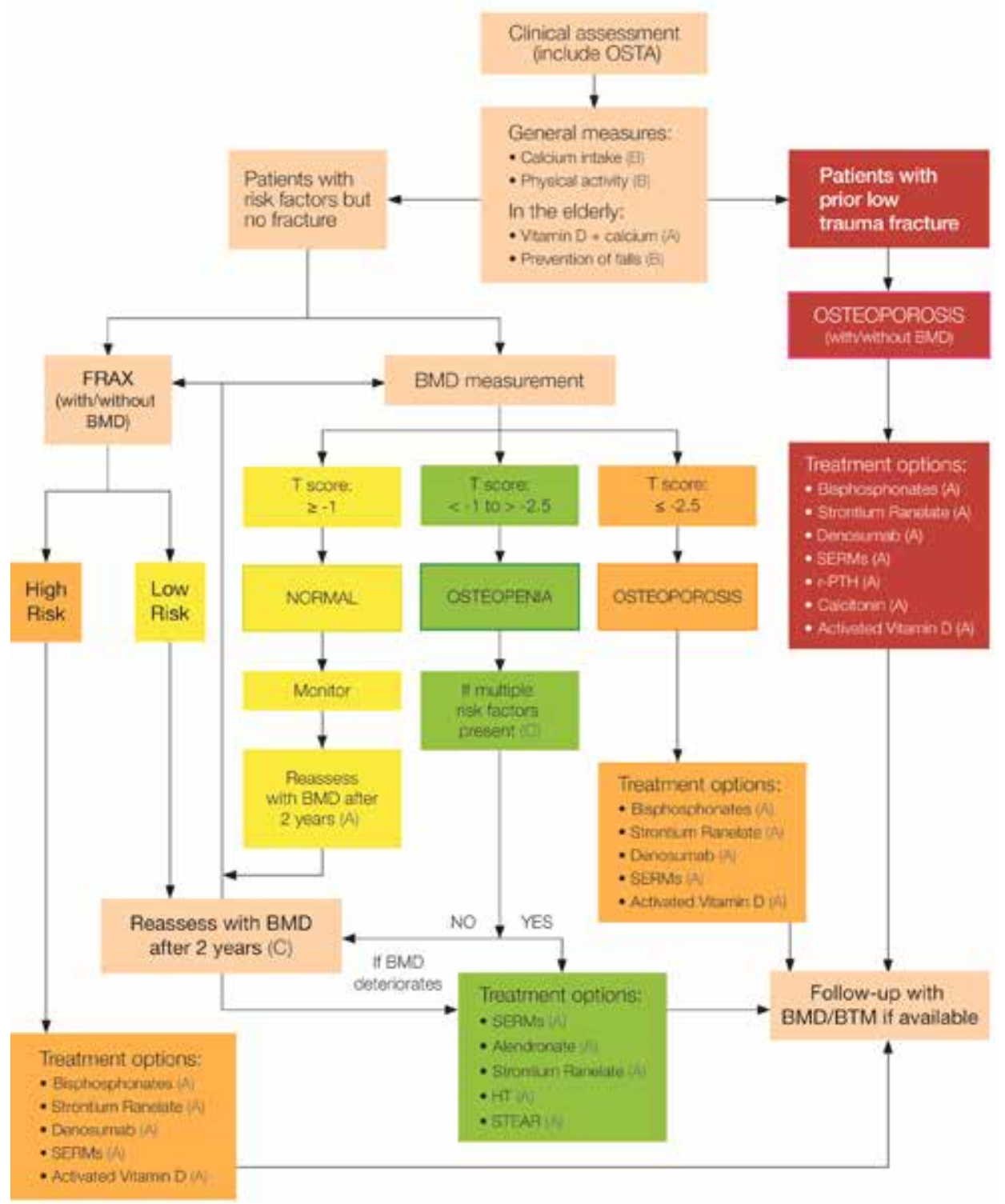

The treatment options found in the algorithm for the management of postmenopausal osteoporosis reflects the order of preference according to current medical evidence. The level of evidence is not a yardstick for comparing relative efficacy. There are few comparative studies between therapeutic agents but the therapeutic aim is for clinical fracture reduction rather then an increase in BMD. Therefore, agents with clinical fracture reduction are ranked higher in the hierarchy of therapeutic choice than agents with only BMD data.

\section{Abbreviations for Figure 1}

OSTA $=$ Osteoporosis Self-Assessment Tool for Asians FRAX $=$ Fracture Risk Assessment Tool BMD $=$ Bone mineral density SERM = Selective estrogen receptor modulator r-PTH $=$ Recombinant parathyroid hormone $\mathrm{HT}=$ Hormone therapy STEAR $=$ Selective tissue estrogenic activity regulator BTM = Bone turnover markers
Grade A Recommendation = Requires at least one randomized controlled trial as part of the body of literature of overall good quality and consistency addressing the specific recommendation.

*The vertebral fracture reduction was shown with the $2.5 \mathrm{mg}$ daily oral dosing. The currently licensed ibandronate dose of $150 \mathrm{mg} / \mathrm{month}$ has been shown to be non-inferior to the $2.5 \mathrm{mg}$ daily dose in terms of BMD gain and bone marker suppression. 
The choice of treatment should be individualised to the patient. For example, hormone therapy (HT) should not be used solely for the prevention of osteoporosis. However, in women who have postmenopausal symptoms, HT can be used up to the age of 60 , and will protect the bones while they are on treatment. Bisphosphonates (BP) have recently been associated with a risk of osteonecrosis of the jaw and atypical femoral shaft fractures (AFF). The absolute risk of these complications arising in patients taking BP for OP is low. AFF have been increasingly recognised in patients on long-term BP therapy. The risk of AFF appears to escalate with increased duration of BP use. However, current evidence has not established a causal association between BP and AFF. It is recommended to evaluate the efficacy of BP therapy after 3-5 years. If a lack of efficacy is noted, i.e. significant deterioration of $\mathrm{BMD}$, or recurrent low trauma fracture occurs, reevaluation is required to exclude secondary causes of OP and to check drug compliance. If both have been excluded, BP can either be continued or an alternative therapy can be considered (i.e. anabolic therapy). When prescribing BP for longer than 5 years, evaluation of the need for continued BP therapy is recommended. In patients with a low risk of fracture, consider a drug holiday. In those with evidence of AFF, BP therapy should be discontinued. In those with a high risk of fracture, consider continuing BP therapy up to 10 years.

\section{Acknowledgement}

Details of the evidence supporting these recommendations can be found in the Clinical Guidance on the Management of Osteoporosis, 2012 , available on the following websites: Ministry of Health Malaysia: http:// www.moh.gov.my/ and Academy of Medicine: http://www.acadmed.org.my/. Corresponding organisation: CPG Secretariat, Health Technology Assessment Section, Medical Development Division, Ministry of Health Malaysia \& contactable at htamalaysia@moh. gov.my 